patients, those receiving tritherapies showed increased risk of structural brain abnormalities $(\mathrm{p}=0.001)$ or SLC2A1 mutations $(\mathrm{p}=0.001)$ but fewer myoclonic features $(\mathrm{p}=0.031)$ and worse seizure-free survival $(\mathrm{p}=0.047)$ than those treated with mono-and biotherapy. Children with NP-EOAE had an increased risk of relapse during follow-up compared to P-EOAE patients. (Agostinelli S, Accorsi P, Beccaria F, et al. and on behalf of the Societa Italiana Neurologia Pediatrica Collaborative Working Group. Epilepsia 2013 Oct;54(10):1761-70). (Response: Dr Alberto Verrotti, Department of Pediatrics, University of Chieti, Italy. E-mail: averrott@unich.it).

COMMENT. Children $<3$ years old with early onset TAS who meet the modified Panayiotopoulos's criteria for childhood absence epilepsy (CAE) have a good prognosis, whereas those not meeting the criteria have an increased risk of relapse at long-term follow-up.

Panayiotopoulos's definition of CAE (Panayiotopoulos CP. Epilepsia 2008 Dec;49(12):2131-9) is abridged as follows: 1) age at onset between 4 and 10 years (modified to within the first 3 years for this study); 2) normal neurologic exam; 3) brief (4-20 sec) and frequent (many per day); 4) EEG 3-4 Hz spike and slow-wave complexes. Exclusion criteria include 1) other types of seizure; 2) eyelid myoclonia, perioral myoclonia, head and limb myoclonic jerks; 3) EEG polyspikes; 4) photic precipitation of clinical seizures.

\title{
TRANSITION FROM PEDIATRIC TO ADULT HEALTH CARE FOR DRAVET SYNDROME PATIENTS
}

Investigators from University Hospital of Rennes; Necker Hospital, Paris; and University Paris Descartes, France used a questionnaire to study the transition and transfer from pediatric to adult health-care system in patients with Dravet syndrome and their families. The diagnosis of Dravet syndrome was made during the first 2 years and was followed by a long follow-up in the pediatric health-care. A response rate of $85 \%$ was obtained from 60 families, and $61 \%$ experienced a transfer. Positive factors for a smooth transition included 1) the quality of transition preparation $(p<.000001), 2)$ a longer duration of follow-up by the same child neurologist $(p<.001), 3)$ the availability of the child neurology staff $(\mathrm{p}<.01), 4)$ transfer into adult health-care after the age of 18 $(\mathrm{p}<.01)$, and 5) a stable medical condition before transfer $(\mathrm{p}<.05)$. The age of transfer (18.7 +/ 4 years) was close to the legal age of adulthood, and the association of mental retardation with severe epilepsy had little impact on transfer age. All families reported a positive experience in the pediatric health care system. Child neurologists were considered as welcoming, available, and helpful. Almost all patients transferred reported no gap in the process. Their experience in the adult health-care system was similar to pediatric care. Only $9 \%$ patients contacted their child neurologist after the transfer, and $79 \%$ continued follow-up with the same neurologist. Preparation for transfer began an average of 1 year before transition, which is shorter than that generally recommended for chronic illnesses. (Kuchenbuch M, Chemaly N, Chiron C, Dulac O, Nabbout R. Transition and transfer from pediatric to adult health care in epilepsy: A families' survey on Dravet syndrome. Epilepsy Behav 2013 Oct;29(1):161-5). (Response: Dr Rima Nabbout, Hospital Necker Enfants Malades, Paris. E-mail: rimanabbout@yahoo.com). 
COMMENT. A Canadian study considering strategies for transitioning to adult care for youth with Lennox-Gastaut syndrome and related disorders (Camfield PR, et al. Epilepsia 2011 Aug;52 Suppl 5:21-7) found that an adult practitioner took less time with the patient and family, and the adult provider was not familiar with the medical disorder. A survey of 133 symposium attendees indicates much dissatisfaction with the process of transition, especially for patients with intellectual handicap. Suggestions to improve transition include identifying a willing adult service, a multidisciplinary approach, adolescent clinics, and attention to vocational training and/or special education.

\section{MOVEMENT DISORDERS}

\section{HYPEREKPLEXIA, APNEAS, DEVELOPMENTAL DELAY, AND GENETIC CORRELATIONS}

Investigators at Swansea University and other centers in the UK, Australia, and Belgium studied the genotype-phenotype correlations in 97 individuals with a clinical diagnosis of hyperekplexia; 61 cases had mutations in GLRA1, 24 cases in SLC6A5 and 12 in GLRB. All gene-positive cases presented in the neonatal period and clonazepam was effective treatment in $95 \%$. Hyperekplexia is a predominantly recessive inheritance, and is dominant in 16\%. In 35 gene-negative cases, presentation was after the first month of life.

The characteristic symptoms of hyperekplexia are 'stiffness, startles and stumbles.' In addition, 50 of 89 patients had apnea attacks and 47 of 92 were developmentally delayed. Recurrent infantile apneas occurred more frequently in patients with SLC6A5 mutations than in those with GLRA1 mutations. Developmental delay occurred more frequently in patients with GLRB and SLC6A5 mutations than in those with GLRA1 mutations; $92 \%$ of GLRB cases had a mild to severe delay in speech acquisition. The developmental delay especially in speech may represent failure of developmental neural networks or migration defects. (Thomas RH, Chung S-K, Wood SE, et al. Genotype-phenotype correlations in hyperekplexia: apnoeas, learning difficulties and speech delay. Brain 2013 Oct;136(Pt 10):3085-95). (Response: Dr Rhys H Thomas, Institute of Life Science, College of Medicine, Swansea University, Singleton Park, Swansea, SA2 8PP. E-mail: Rhys.Thomas@swansea.ac.uk).

COMMENT. Hyperekplexia was first described by Kok O, and Bruyn GW (Lancet 1962;279(7243):1359) in 29 members of one family and occurred as a dominant autosomal transmission. Hypertonia is present at birth and becomes less pronounced during the first year of life but later leads to repeated falls. The name "hereditary stiff baby syndrome" was used by Lingam S, et al. (Am J Dis Child 1981 Oct;135(10):90911). The child has a fixed stare and an expression of anxiety. Hypertonia diminishes during sleep and increases with the slightest psychic or tactile stimulus. Nose tapping elicits the hyperekplexic response and is included in the neonatal exam of infants at risk. Attacks of hypertonia that involve the respiratory muscles can lead to apneas that endanger life. The EMG shows persistent activity abolished by diazepam or clonazepam; the EEG is normal. 